\title{
Erratum to: The Minimal Speed of Traveling Fronts for the Lotka-Volterra Competition System
}

\author{
Jong-Shenq Guo • Xing Liang
}

Received: 23 October 2013 / Published online: 12 November 2013

C) Springer Science+Business Media New York 2013

\section{Erratum to: J Dyn Diff Equat (2011) 23:353-363 DOI 10.1007/s10884-011-9214-5}

We study in [1] the minimal speed of traveling front for the following Lotka-Volterra competition system:

$$
\begin{gathered}
u_{t}=u_{x x}+u(1-u-k v), \quad x, t \in \mathbb{R}, \\
v_{t}=d v_{x x}+r v(1-v-h u), \quad x, t \in \mathbb{R},
\end{gathered}
$$

where $d>0, r>0,0<k<1<h$. The main purpose is to derive some conditions on the parameters $h, k, r, d$ for which the so-called linear determinacy ([3]) holds for the system (0.1)-(0.2). For this, we first study the following sequence of discretized problems:

$$
\begin{gathered}
\left(u_{j}^{\tau}\right)^{\prime}(t)=\mathcal{D}_{2}\left[u_{j}^{\tau}\right](t) / \tau^{2}+u_{j}^{\tau}(t)\left[1-u_{j}^{\tau}(t)-k v_{j}^{\tau}(t)\right], j \in \mathbb{Z}, t \in \mathbb{R}, \\
\left(v_{j}^{\tau}\right)^{\prime}(t)=d \mathcal{D}_{2}\left[v_{j}^{\tau}\right](t) / \tau^{2}+r v_{j}^{\tau}(t)\left[1-v_{j}^{\tau}(t)-h u_{j}^{\tau}(t)\right], j \in \mathbb{Z}, t \in \mathbb{R},
\end{gathered}
$$

for $\tau>0$, where $\mathcal{D}_{2}\left[w_{j}\right]:=\left(w_{j+1}-w_{j}\right)+\left(w_{j-1}-w_{j}\right)$. Then, by passing the limit for $\tau \rightarrow 0^{+}$, we derive the linear determinacy for the continuous system (0.1)-(0.2). A careful check of the proof of [1, Theorem 1], we have the linear determinacy for the discrete problem (0.3)-(0.4) if $(h, k, r, d) \in B_{1} \cup B_{2}$, where

The online version of the original article can be found under doi:10.1007/s10884-011-9214-5.

J.-S. Guo

Department of Mathematics, Tamkang University, Tamsui, Taipei 25137, Taiwan

e-mail: jsguo@mail.tku.edu.tw

X. Liang $(\varangle)$

Wu Wen-Tsun Key Laboratory of Mathematics and Department of Mathematics, University of Science and Technology of China, Hefei, People's Republic of China e-mail: xliang@ustc.edu.cn 


$$
\begin{aligned}
& B_{1}:=\left\{d \in\left(0, d_{*}\right], 1<h \leq 1 / k, r>0\right\}, \\
& B_{2}:=\left\{d \in\left(0, d_{*}\right], h>1 / k, 0<r \leq \frac{d_{*}-d}{d_{*}-1} \frac{1-k}{h k-1}\right\}
\end{aligned}
$$

for some constant $d_{*}>2$. However, the constant $d_{*}$ depends on $\tau$ and one can prove that $d_{*}(\tau) \downarrow 2$ as $\tau \rightarrow 0^{+}$([2]). Therefore, [1, Theorem 2] has to be corrected. In fact, we can only re-produce the result of [3] (except when $r=(2-d)(1-k) /(h k-1)$ for $d \in(0,1))$. Although our method is very indirect, it can be used in other similar problems (see, e.g., [2]). Moreover, we also obtain the linear determinacy for the corresponding discrete problem.

\section{References}

1. Guo, J.-S., Liang, X.: The minimal speed of traveling fronts for the Lotka-Volterra competition system. J. Dyn. Diff. Equat. 23, 353-363 (2011)

2. Guo J.-S., Wang Y., Wu C.-H., Wu C.-C.: The existence of traveling wave solutions for a three species competition system (preprint)

3. Lewis, M.A., Li, B., Weinberger, H.F.: Spreading speed and linear determinacy for two-species competition models. J. Math. Biol. 45, 219-233 (2002) 